\title{
A NEW DYNAMIC DEPLOYMENT APPROACH BASED ON WHALE OPTIMIZATION ALGORITHM IN THE OPTIMIZATION OF COVERAGE RATES OF WIRELESS SENSOR NETWORKS
}

\author{
Recep ÖZDA $\breve{G}^{* 1}$, Murat CANAYAZ ${ }^{2}$ \\ ${ }^{* 1}$ Van Yüzüncü Y1l University, Department of Computer Engineering, Van, TURKEY \\ ${ }^{2}$ Van Yüzüncü Y1l University, Department of Computer Engineering, Van, TURKEY \\ ${ }^{*}$ Corresponding author; E-mail: rozdag@yyu.edu.tr
}

The dynamic deployments of Wireless Sensor Networks refer to the process of determining the location of the networking sensors in the region of interest after initial deployment. In this process, the fact that the dynamic deployments of sensors is effectively made plays a significant role in increasing the coverage rates of WSNs. The optimization of the coverage rates of Wireless Sensor Networks indicates that the targets in the region of interest are optimally covered by deployed mobile sensors and ultimately these targets can be monitored by the sensors. Therefore, the monitoring of the whole area in Wireless Sensor Network's military and civilian applications is possible by the optimum placement of randomly deployed sensors in the region of interest. In this study, a new dynamic deployment approach was developed based on the current meta-heuristic Whale Optimization Algorithm to find a solution to the problem of dynamic deployment of sensors. In order to solve the area coverage problem of Wireless Sensor Networks, the dynamic deployments of mobile nodes, the initial deployment of which was made randomly, was made by the developed approach using the Binary Detection Model. This approach was compared with the Maximum Area Detection Algorithm based on Electromagnetism-Like in the literature, and the performance of Wireless Sensor Network at coverage rates was measured. Simulation results have demonstrated that the approach developed for the area coverage problem is more effective and can be suggested with respect to the number of mobile sensors deployed and the reached coverage rates of the network.

Key words: Wireless Sensor Networks, Area Coverage Problem, Sensor Dynamic Deployment, Binary Detection Model, Whale Optimization Algorithm 


\section{Introduction}

Wireless Sensor Networks (WSNs) are used in various applications such as the applications requiring national security like environmental monitoring and monitoring of critical environments, health practices and determination of disastrous situations. WSNs are formed when sensor nodes in a deployed structure with the features of sensing, computing, communicating and moving come together [1]. Unlike static nodes, mobile nodes can change their initial positions in the network due to their ability to move. The coverage rates of WSNs can be optimized by ensuring that the nodes effectively cover the region of interest with the deployment strategies in the literature [2] developed for mobile nodes. Therefore, the dynamic deployments of mobile nodes in the area should be made effectively for the optimal coverage of the region of interest.

WSNs have some restrictions that prevent Quality of Service (QoS). These restrictions consisting of the limited powers, limited communication and computing capabilities and limited bandwidths of sensor nodes bring along some problems in accordance with the purposes of WSNs. One of the most important problems is the effective deployment of nodes that determine the coverage rate performance of the WSNs in the area [3].

Sensors are usually deployed in the region of interest by making deterministic or stochastic (random) deployments. It is not possible to perform sensor deployment manually in many potential working environments such as remote areas, natural disaster areas and urban areas [4]. These nodes can be deployed by airplane. However, when this technique, which is known as the random deployment method, is used, the actual landing positions of nodes cannot be controlled if there are obstacles or wind such as trees and buildings in the environment. As a result, the coverage requirement targeted in the node deployment practices performed with random deployment methods cannot be met [5]. The coverage rate of WSN can be optimized by meeting the coverage requirements required for the targets in the area with the deterministic deployment of nodes. In addition, the fact that the targets can be covered in the applications that require the monitoring of military and civilian environments can be provided by the planned and balanced deployment of randomly deployed nodes in the region of interest. Therefore, the active deployment of the sensors in the area is possible by performing the deterministic deployment of nodes. The node deployment made by using meta-heuristic algorithms [6,7], which is also the subject of this article, serves as an example of the deterministic deployment in WSNs.

While the coverage of the entire region of interest in WSNs is expressed as the area coverage problem, the coverage of only some targets in the area is expressed as the target coverage problem. Area coverage requires that all targets in the area are covered by at least one sensor node for the maximum coverage of a monitored area on the network. Target coverage requires that each target point should be covered by a sufficient number (at least $k$ [3]) of sensors in order for WSNs to meet coverage requirements for QoS. The coverage rates of the region of interest are calculated on the basis of Binary Detection Model or the Probabilistic Detection Model. The coverage rates of the WSN are calculated by assuming that there is no environmental uncertainty that prevents node deployments in the Binary Detection Model and by assuming that there are environmental uncertainties such as wind in the Probabilistic Detection Model [8]. To find a solution to the area coverage problem by means of Binary Detection Model, all targets in the area should be covered by at least one sensor. However, the coverage of a target by more than one sensor does not increase the network's coverage rate and causes the lifetime of the network to decrease by increasing the energy consumption of sensors. Therefore, the coverage 
rate of the network should be optimized by using as few sensors as possible, and the sensors should be ensured to cover the targets in a balanced manner by preventing a target point from being covered by more than one sensor. Thus, the over-concentration of sensors on certain targets is prevented and also optimum numbers of targets are ensured to be covered.

In the literature, many studies have been carried out to optimize the coverage rate of the network in the area coverage problem. Some of these studies were carried out on the basis of heuristic algorithms [8-10], and some of them were carried out with certain strategies and techniques based on various regular deployment topologies [11-13]. Optimization algorithms have been used by most researchers in dynamic deployment optimization of WSNs since they give quite good results in the deployment of sensors forming the WSNs. Virtual Force (VF) [14] algorithm is one of the important algorithms that optimize the coverage rate of the network. The VF algorithm gave positive results in the deployment optimization of WSNs consisting of mobile sensor nodes [15]. A distributed strategy that increases the coverage rate of the network has been proposed by decreasing the distance between adjacent sensors with the Particle Swarm Optimization (PSO) [16], which is a population-based heuristic optimization algorithm. In addition, VFCPSO [17] consisting of the combination of the Co-evolutionary Particle Swarm Optimization (CPSO) and the VF algorithm has been proposed to perform an effective dynamic deployment of nodes. With the purpose of optimizing the coverage rates of WSNs, the meta-heuristic Artificial Bee Colony (ABC) algorithm was taken as a basis and applied to mobile sensors [7] with the Binary Detection Model and to static sensors [18] with the Probabilistic Detection Model, and it was proposed as a new approach to the dynamic deployment of WSNs. By using the Electromagnetism-Like (EM) algorithm, which is based on the pushing and pulling mechanism of charged particles in the solution space, effective dynamic deployment of sensors was made and the coverage rates of WSNs were optimized. In this process, the Optimal Sensor Detection Algorithm based on EM (OSDA-EM) was primarily developed, and it was applied to mobile [19] and static [20] sensors. Then, the Maximum Area Detection Algorithm based on EM (MADA-EM) [21] was developed through a new objective function computational technique, and it was only applied to mobile sensors and optimum results were obtained. MADA-EM is a new dynamic deployment approach developed with the aim of covering maximum numbers of destinations by updating the locations of the mobile sensors.

In this paper, the Maximum Area Detection Algorithm based on Whale Optimization Algorithm [22] (MADA-WOA) was developed in order to optimize the coverage rate of WSNs based on the Binary Detection Model by performing the dynamic deployment of the mobile sensors that are deployed randomly on a grid-based deployment area. With MADA-WOA, dynamically deployed sensors in the region of interest were aimed to cover maximum numbers of Grid (target) points.

In this study, the dynamic deployment problem of sensor nodes is identified in Section 2, and meta-heuristic WOA is explained in Section 3. The algorithmic structure of MADA-WOA developed for the effective deployment of the sensors is presented in Section 4. The experimental findings obtained in the MATLAB ${ }^{\circledR}$ environment were discussed in comparison with MADA-EM in Section 5, and the results obtained are presented in Section 6.

\section{Dynamic Deployment Problem of Sensors}

In this study, a WSN which is composed of $S=\left\{s_{1}, s_{2}, \ldots, s_{n}\right\}$ sensor node set and in which all nodes are mobile was defined on $A$ square area which is a two dimensional solution space. The sensing interval of each $s_{i}$ node in the $S$ set was defined as a central circle of this node with a detection radius 
of $r_{i}$. By assuming that the distance between each Grid point $\left(P^{(x, y)}\right)$ in $A$ was 1 meter, the deployment of $S$ node set on $(x, y)$ coordinate $A$ area was performed randomly at the beginning. The coverage status of these targets for $P^{(x, y)}$ in $A$ by $s_{i}$ node was defined as $\operatorname{cs}\left(P, s_{i}\right)$.

\subsection{Definition 1: upper and lower bound of the region of interest}

The locations of the nodes that will be randomly and dynamically deployed are defined according to Eq. (1) provided that $A$ is between the upper and lower bounds.

$$
\begin{gathered}
\forall x \in A, \\
\left\{x \in R^{n} \mid l_{j} \leq x_{j} \leq u_{j}: l_{j}, u_{j} \in R, j=1,2, \ldots, n\right\}
\end{gathered}
$$

In the given equation, $n$ represents the solution space dimension, $l_{j}$ and $u_{j}$ represent the upper and lower bounds of the region of interest, and $x_{j}$ represents the locations of the nodes in the area.

\subsection{Definition 2: coverage status of target points}

According to Binary Detection Model, if the $d\left(s_{i}, P\right)$ Euclidean distance (Eq. (2)) between the $s_{i}$ node and $P^{(x, y)}$ target point in $A$ is smaller than $r_{i}$ or equal to it (Eq. (3)), this target is accepted to be covered by $S_{i}$ node [19].

$$
\begin{gathered}
\forall P^{(x, y)} \in A, \\
d\left(s_{i}, P\right)=\sqrt{\left(x_{i}-x\right)^{2}+\left(y_{i}-y\right)^{2}} \\
c s\left(P, s_{i}\right)= \begin{cases}1, & \text { if } \quad d\left(s_{i}, P\right) \leq r_{i} \\
0, & \text { otherwise }\end{cases} \\
\mu^{P(x, y)}=\left(\mu_{1}^{P(x, y)}, \mu_{2}^{P(x, y)}, \ldots, \mu_{n}^{P(x, y)}\right), \text { in which the information whether } P^{(x, y)} \text { point was }
\end{gathered}
$$
covered by $S$ set was defined and which is a set consisting of $n$ number of target points provided that it is $1 \leq i \leq n$, was defined. Accordingly, $c s\left(P, s_{i}\right)$ is calculated for $\forall P^{(x, y)} \in A$ by assigning $\mu_{i}^{P(x, y)}=1$ , if $P^{(x, y)}$ is covered and $\mu_{i}^{P(x, y)}=0$ if it is not covered.

\subsection{Definition 3: coverage rate of the region of interest}

The coverage rate (cr) of the WSN is determined according to Eq. (4) by calculating the total numbers of $P^{(x, y)}$ covered by the nodes in the region of interest.

$$
c r(A)=\frac{\sum_{i=1}^{n} \mu_{i}^{P(x, y)}}{(-l+1)(u+1)}
$$

In the equation given [21], total number of targets in $A$ is determined by multiplying the total Grid lengths of the lower and upper bounds of $A$, provided that it is $\forall P^{(x, y)} \in A$. 


\section{Whale Optimization Algorithm}

The WOA, one of the new meta-heuristic optimization algorithms, was developed by being inspired by the bubble-net hunting strategy used by humpback whales while hunting [22]. This swarmbased algorithm has many unique features such as PSO, ABC and Cricket Algorithm [23].

The humpback whales fed by small fish swarms have their unique hunting strategy. The whales that breathe under the water and form bubble clouds gather their prey this way. They maintain the bubble cloud to the surface of the water and ensure that their prey in this cloud. Thus, they have also hidden themselves. Fig. 1(a) shows a representative picture of hunting methods of humpback whales by bubblenet hunting strategy, Fig. 1(b) shows a real picture of hunting of humpback whales by bubble-net hunting strategy [24]. In the whale optimization algorithm, hunting strategy was modeled in three parts including surrounding the prey, moving towards the prey and seeking for the prey.

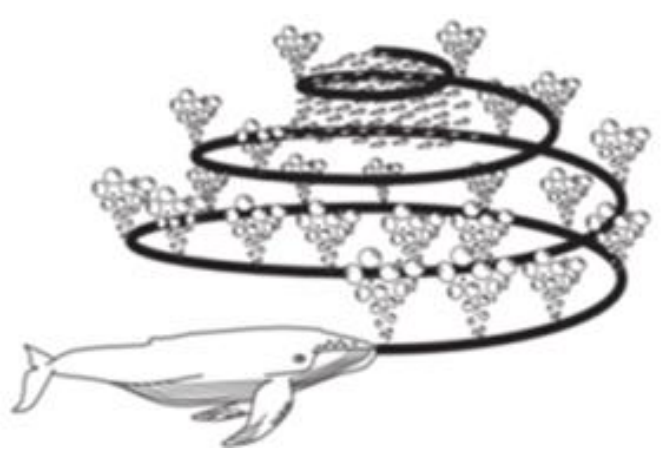

(a)

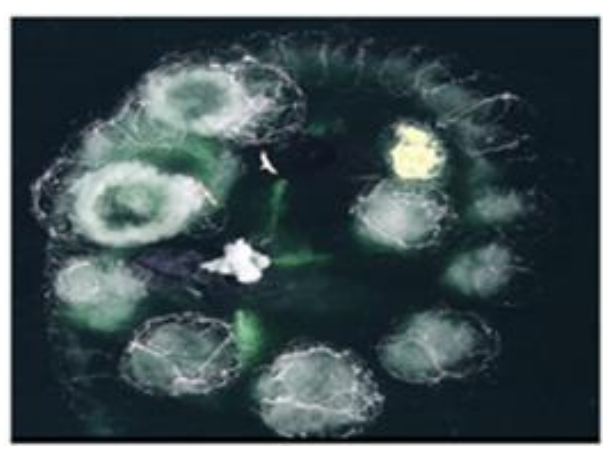

(b)

Fig. 1. (a) Representative and (b) Real pictures of hunting methods of humpback whales by bubble-net hunting strategy.

\subsection{Surrounding the prey}

In the WOA, the prey is accepted as the optimum solution to be reached. Since optimum solution is not known in optimization problems, an attempt to achieve optimum solution using local or global search spaces is made. The seeking process continues until certain criteria are met. Optimum solution is accepted as the best solution reached or a point around it. After the best solution is determined, the locations of the other solutions are updated using the best solution. The mathematical model of the behavior of surrounding the prey is presented in Eq. (5) and Eq. (6).

$$
\begin{gathered}
\vec{D}=\left|\vec{C} \cdot \vec{X}^{*}(t)-\vec{X}(t)\right| \\
\vec{X}(t+1)=\left|\vec{X}^{*}(t)-\vec{A} \cdot \vec{D}\right|
\end{gathered}
$$


In the equation given [22], $t$ represents the instant iteration, $A$ and $C$ represent the coefficient vectors, $X^{*}$ represent the best solution vector.

In Eq. (7) and Eq. (8), $r$ represent a random vector, $a$ represents a vector decreasing from 2 to 0 throughout the iterations [22].

$$
\begin{gathered}
\vec{A}=2 \vec{a} \cdot \vec{r}-\vec{a} \\
\vec{C}=2 \cdot \vec{r}
\end{gathered}
$$

\subsection{Moving towards the prey}

The whales that have find the prey come closer to it in two different ways such as narrowing the circle around the prey and spiral movement in order to move towards their prey. These movements are modeled in Fig. 2. It was thought that it would be possible to narrow the circle around the prey by the method of decreasing the value of $a$ in Eq. (7). The spiral movement of the whales and the location of the best solution are presented in Fig. 2 [22]. Eq. (9) was established by calculating the distance between the target location and the solution candidate for this movement [22].

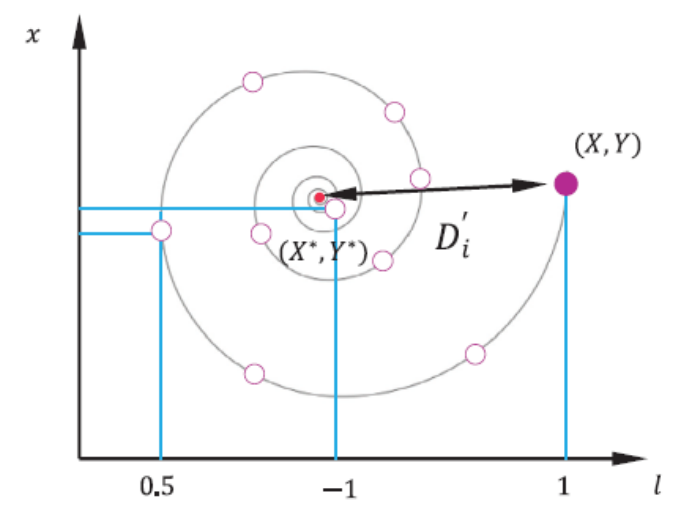

Fig. 2. Spiral movement

$$
\begin{gathered}
\vec{X}(t+1)=\vec{D}^{\prime} \cdot e^{b l} \cdot \cos (2 \pi l)+\vec{X}^{*}(t) \\
\vec{D}^{\prime}=\vec{X}^{*}(t)-\vec{X}(t)
\end{gathered}
$$

In the equation given [22], $b$ represents the logarithmic spiral constant, $l$ represents a random number in the range of $[-1,1]$. In the algorithm, which one of them, spiral movement or linear movement, will be employed is determined by probability $1 / 2$ as it is shown in Eq. (10).

$$
\vec{X}(t+1)= \begin{cases}\left|\vec{X}^{*}(t)-\vec{A} \cdot \vec{D}\right|, & p<0.5 \\ \vec{D}^{\prime} \cdot e^{b l} \cdot \cos (2 \pi l)+\vec{X}^{*}(t), & p \geq 0.5\end{cases}
$$


In the equation given [22], $p$ represents a random number generated in the range of $[0,1]$.

\subsection{Seeking for the prey}

For the global solution, the new locations of the solution candidates are determined around a randomly selected solution candidate instead of the best known point. The mathematical model is presented in Eq. (11) and Eq. (12).

$$
\begin{gathered}
\vec{D}^{\prime}=\vec{C} \cdot \vec{X}_{\text {rand }}-\vec{X} \\
\vec{X}(t+1)=\vec{X}_{\text {rand }}-\vec{A} \cdot \vec{D}
\end{gathered}
$$

In the equation given [22], $X_{\text {rand }}$ represents a randomly selected solution vector. Which one of them, global or local searches, will be performed is decided based on the value of vector $A$. Since the point which is more distant than the best point can be selected when it is $A>1$ or $A<1$ for vector $A$ , these cases are regarded as Global search and are applied by Eq. (11) and Eq. (12).

\section{WOA Based New Dynamic Deployment Approach}

In this study, the dynamic deployment of the nodes was performed by MADA-WOA which was developed with the aim of being able to optimize the coverage rate of the network after the random deployment of the nodes. To be able to achieve this aim, the deployment of the nodes, the locations of which were updated by the WOA, in the optimum location in the area was targeted. The fitness function $(f(x))$ of the nodes was calculated by MADA-WOA, and the coverage of the maximum number of target points in the region of interest was ensured.

The initial deployments of the nodes were made randomly, and they were ensured to be deployed in the region of interest. In this area, a Grid point was randomly selected and defined as the Leader target point. The reason for the defining of this target as Leader is that if $f(x)^{P}$ value calculated for this Grid point, by assuming that there is a node in this target, is $\left(f(x)^{\text {opt }}\right)$ between the range of $f(x)^{\text {upper }}$ and $f(x)^{\text {lower }}$ defined, all mobile nodes in the network are directed towards this Leader target and their locations are updated by WOA. Indeed, the fact that the $f(x)^{P}$ value calculated for this selected Leader target is in the range of $f(x)^{\text {opt }}$ assures that the calculated $f(x)^{S}$ value of the nodes to be deployed around this target could be in the range of $f(x)^{\text {opt }}$. The fact that the $f(x)$ value calculated MADAWOA for both Leader target point and the nodes is optimum makes it possible to reach the node that covers a maximum number of Grid points. In the event that if $f(x)$ values are not in the range of $f(x)^{\text {opt }}, f(x)^{\text {lower }}$ value is updated by decreasing it in the defined range, and an attempt to reach optimum solution is made by randomly reselecting a Leader target point. If the calculated $f(x)^{S}$ value of any of the nodes reaches the $f(x)^{\text {opt }}$ range after the node deployment performed with WOA, this node is determined as the Optimum Sensor $\left(s^{\text {opt }}\right)$. Therefore, in the next iterations, it is ensured that the Grid points covered by $s^{o p t}$ are reserved only for $s^{o p t}$ without changing it location, and it is targeted that these Grid points will not be covered again. As a result, the number of targets covered increases as the number of $s^{o p t}$ increases in iterations, the reserved Grid points in the area are ensured to be covered by a minimum number of $s^{o p t}$, and the coverage rate of WSN is optimized. 


\section{Experimental Findings}

Along with the MADA-WOA, simulation studies of MADA-EM in the literature were conducted using the MATLAB ${ }^{\circledR}$ program. The simulation results obtained to determine the performance of the mobile nodes in the related area in their dynamic deployment by MADA-WOA were compared with MADA-EM [21].

The common parameters used for the algorithms compared in this study are presented in Tab. 1 . In the simulations, the coverage rate of WSN was calculated by determining the number of targets of the mobile node in the range of 20 - 100 with a detection radius of 7 meters in the area of $10000 \mathrm{~m}^{2}$ by employing a maximum of 100 iterations. Five independent simulation studies were conducted to determine the stability of the algorithms on WSN's coverage rates.

Table 1. Common parameters of the compared algorithms

\begin{tabular}{l|l}
\hline \multicolumn{1}{c|}{ Parameter } & \multicolumn{1}{c}{ The value or range } \\
\hline Area size & $100 \times 100 \mathrm{~m}\left(10000 \mathrm{~m}^{2}\right)$ \\
Number of Grid in Area & 10201 \\
Grid distance & $1 \mathrm{~m}$ \\
Number of mobile sensor & $20-100$ \\
Sensing radius of sensor & $7 \mathrm{~m}$ \\
Maximum number of iteration & 100 \\
Fitness function's update range & 5 \\
Number of simulation & 5 \\
\hline
\end{tabular}

The results obtained by the independent simulation studies are presented in Tab. 2. When the average coverage rates of the algorithms calculated according to the number of mobile nodes deployed were compared, more optimum results were achieved with MADA-WOA (Fig. 3). The fact that the standard deviation calculated with MADA-WOA is always lower indicates that the coverage rates of WSN are closer to each other in each simulation study performed with the algorithm developed. It was also determined that the average coverage rate of $78 \%$, which was achieved in the dynamic deployment of 100 mobile nodes by MADA-EM, could be reached by MADA-WOA only when 60 mobile nodes were deployed. Therefore, it can be said that the MADA-WOA which was developed in the calculation of coverage rates of WSN is more optimum and a more stable approach compared to other algorithm.

Table 2. Coverage rates calculated by the algorithms based on the number of nodes

\begin{tabular}{lcccccccc}
\hline \multirow{2}{*}{$\begin{array}{l}\text { Number } \\
\text { of }\end{array}$} & \multicolumn{9}{c}{ MADA-WOA } \\
\cline { 2 - 9 } $\begin{array}{l}\text { Mobile } \\
\text { Nodes }\end{array}$ & $\begin{array}{c}\text { Maximum } \\
\text { Coverage } \\
\text { Ratio }\end{array}$ & $\begin{array}{c}\text { Minimum } \\
\text { Coverage } \\
\text { Ratio }\end{array}$ & $\begin{array}{c}\text { Average } \\
\text { Coverage } \\
\text { Ratio }\end{array}$ & $\begin{array}{c}\text { Standard } \\
\text { Deviation }\end{array}$ & $\begin{array}{c}\text { Maximum } \\
\text { Coverage } \\
\text { Ratio }\end{array}$ & $\begin{array}{c}\text { Minimum } \\
\text { Coverage } \\
\text { Ratio }\end{array}$ & $\begin{array}{c}\text { Average } \\
\text { Coverage } \\
\text { Ratio }\end{array}$ & $\begin{array}{c}\text { Standard } \\
\text { Deviation }\end{array}$ \\
\hline 20 & 29.22 & 29.18 & 29.21 & 0.01 & 29.13 & 28.67 & 29.01 & 0.19 \\
40 & 57.09 & 56.89 & 56.97 & 0.07 & 56.37 & 53.52 & 54.58 & 1.26 \\
60 & 79.00 & 78.27 & 78.55 & 0.30 & 68.15 & 64.20 & 66.14 & 1.52 \\
80 & 91.34 & 89.98 & 90.64 & 0.60 & 77.61 & 73.68 & 74.95 & 1.55 \\
100 & 95.68 & 94.83 & 95.47 & 0.36 & 81.56 & 75.91 & 78.29 & 2.07 \\
\hline
\end{tabular}




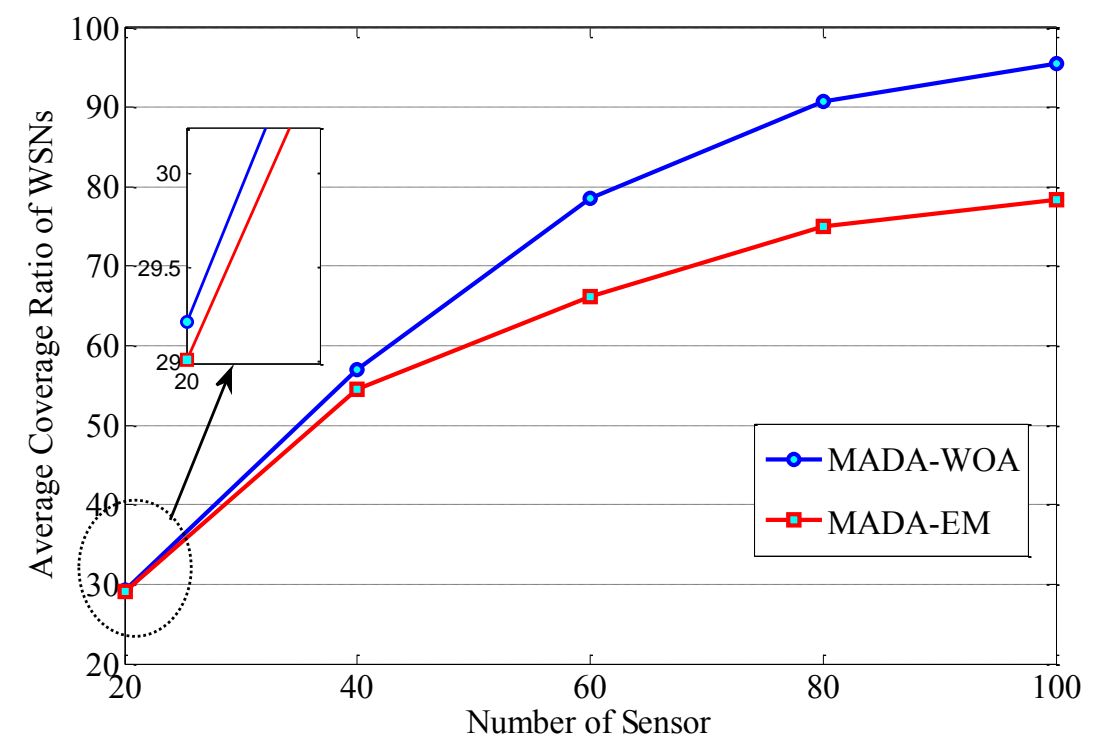

Fig. 3. WSN's coverage rate change graph according to algorithms

The mobile sensors that were dynamically distributed by MADA-WOA and MADA-EM were statically deployed to the region of interest from the moment that they were optimum. The relevant simulation study is terminated without reaching maximum number of iterations because iteration will not be performed again when all nodes deployed in both algorithms are optimum. Therefore, the fact that the average number of iterations in the simulation studies is very close or equal to maximum number of iterations means that iteration has been terminated before all dynamically deployed mobile nodes in the area cannot be deployed in the optimum location. According to the simulation results presented in Tab. 3, when only 20 nodes were deployed by MADA-EM, all nodes were deployed in the optimum location after an average of 52 iterations. Moreover, it was determined that all nodes deployed in the range of $40-100$ could not be deployed in the optimum location after 100 iterations. However, in MADA-WOA, all mobile nodes deployed in the range of 20 - 60 were deployed in the optimum location without even employing for 100 iterations. Furthermore, it was determined that the average optimum number of nodes reached by MADA-WOA was optimum compared to the other algorithm according to the number of nodes deployed. As the number of optimum nodes increases, the coverage rate of the region of interest increases directly proportional (Tab. 2). Therefore, when the average optimum number of nodes reached according to the number of the deployed mobile nodes were taken as basis, mobile node in the range of $20-80$ was deployed by the developed MADA-WOA, and it was determined that it was more quickly converged in reaching the optimum number of node compared to other algorithm.

Table 3. Optimum number of nodes reached in the algorithms based on the number of sensors

\begin{tabular}{lcccc}
\hline \multirow{2}{*}{$\begin{array}{l}\text { Number } \\
\text { Mobile }\end{array}$} & \multicolumn{2}{c}{ MADA-WOA } & \multicolumn{2}{c}{ MADA-EM } \\
\cline { 2 - 5 } Nodes & $\begin{array}{c}\text { Average Number } \\
\text { of Iterations }\end{array}$ & $\begin{array}{c}\text { Average Optimum } \\
\text { Number of Nodes }\end{array}$ & $\begin{array}{c}\text { Average Number } \\
\text { of Iterations }\end{array}$ & $\begin{array}{c}\text { Average Optimum } \\
\text { Number of Nodes }\end{array}$ \\
\hline 20 & 35.40 & 20.00 & 52.20 & 20.00 \\
40 & 69.80 & 40.00 & 100.00 & 37.80 \\
60 & 92.60 & 60.00 & 100.00 & 43.40 \\
\hline
\end{tabular}




\begin{tabular}{lcccc}
\hline 80 & 99.20 & 79.40 & 100.00 & 45.80 \\
100 & 100.00 & 89.00 & 100.00 & 45.40 \\
\hline
\end{tabular}

In order to show that MADA-WOA performed an effective dynamic deployment, the deployments of 80 mobile nodes, the dynamic deployments of which were performed by the algorithms, in the region of interest are graphically presented in Fig. 4.

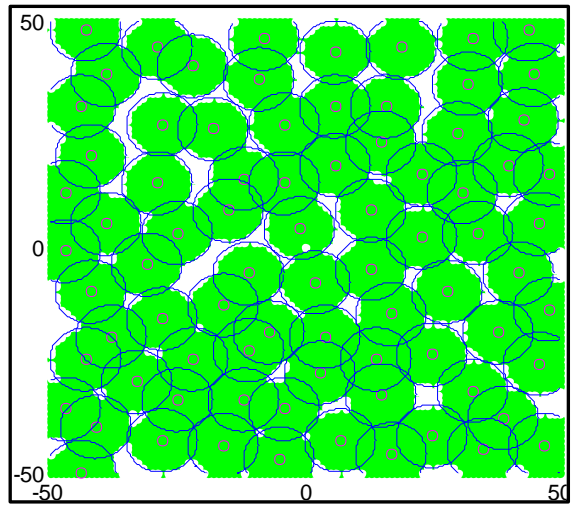

(a)

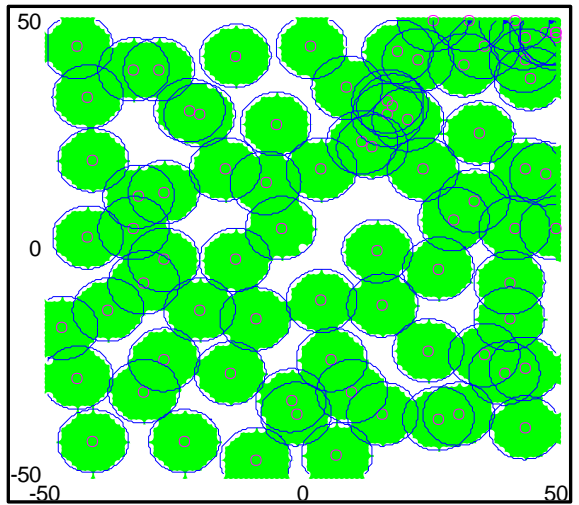

(b)

Fig. 4. Graph of optimum coverage rates of $91.34 \%$ with (a) MADA-WOA and $77.61 \%$ with (b) MADA-EM by dynamically deployed 80 mobile nodes

\section{Conclusions}

In this study, in order to optimize the coverage rate of the WSN, MADA-WOA was developed to be able to perform the effective dynamic deployment of the nodes, the initial deployment of which was performed randomly. The simulation results calculated to show that the effective dynamic deployment of the nodes in the region of interest could be performed with this algorithm were compared with the MADA-EM in the literature. According to these results, it was determined that the standard deviation of the coverage rates calculated by MADA-WOA was minimum compared to the other algorithm and thus MADA-WOA was more stable, that the coverage rates calculated by MADA-WOA were more optimum as the optimum number of mobile nodes reached were increased, and that the mobile nodes deployed by the MADA-WOA were more quickly converged to the region of interest and were deployed in the optimum location. In addition, it was also determined that the coverage rate which was reached when 60 mobile nodes were deployed by MADA-WOA could only be reached when 100 mobile nodes were deployed by MADA-EM. Therefore, due to the fact that fewer nodes are deployed, WSN's lifetime will increase since the energy consumption of the nodes will also be optimized.

In conclusion, MADA-WOA was proposed to perform the effective dynamic deployment of the nodes because of the fact that optimum coverage rates were more stably reached with the algorithm developed, that the deployed nodes converged faster while being deployed in the optimum location and that the lifetime of WSN could also be optimized by performing the deployment of a smaller number of mobile nodes in the region of interest.

In the next study, this algorithm proposed is also planned to be applied to the probabilistic detection model. 


\section{Acknowledgment}

This project was supported by Van Yüzüncü Y1l University Scientific Research Projects Coordination Unit (YYÜBAP) under project number FBA-2017-5831.

\section{Nomenclature}

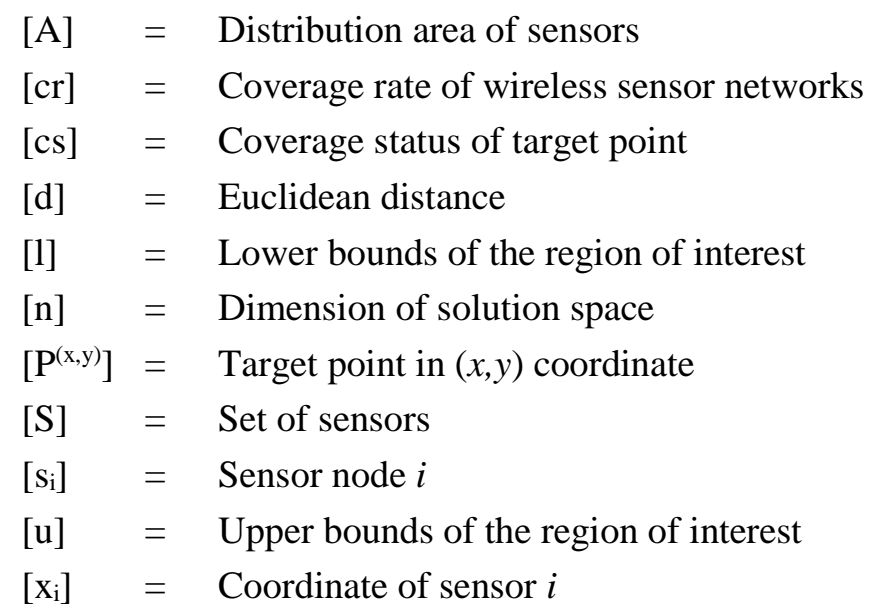

\section{References}

[1] Yıldırım, F., Özdemir, S., Improving Coverage in Wireless Sensor Networks Using Multiobjective Evolutionary Algorithms, Journal of the Faculty of Engineering and Architecture of Gazi University, 30 (2015), 2, pp. 143-153.

[2] Clouqueur, T., Phipatanasuphorn, V., Ramanathan, P., Saluja, K.K., Sensor Deployment Strategy for Target Detection, Proceedings, 1st ACM International Workshop on Wireless Sensor Networks and Applications, Atlanta, Georgia, USA, 2002, pp. 42-48

[3] Özdağ, R., The Solution of the k-coverage Problem in Wireless Sensor Networks, Proceedings, 24th Signal Processing and Communications Applications Conference, Zonguldak, Turkey, 2016, pp. 873-876

[4] Wang, G., Cao, G., La Porta T.F., Movement-assisted Sensor Deployment, IEEE Transactions On Mobile Computing, 5 (2006), 6, pp. 640-652, doi: 10.1109/TMC.2006.80

[5] Özdăg, R., Optimization of Target Q-Coverage Problem for QoS Requirement in Wireless Sensor Networks, Journal of Computers, 13 (2018), 4, pp. 480-489, doi: 10.17706/jcp.13.4 480-489

[6] Qi, G.P., Song, P., Li, K.J., Blackboard Mechanism Based Ant Colony Theory for Dynamic Deployment of Mobile Sensor Networks, Journal of Bionic Engineering, 5 (2008), 3, pp. 197-203.

[7] Öztürk, C., Karaboğa, D., Görkemli. B., Artificial bee colony algorithm for dynamic deployment of wireless sensor networks, Turk J. Elec. Eng. \& Comp. Sci., 20 (2012), 2, pp. 255-262.

[8] Özdağ, R., Realization of Optimization of Wireless Sensor Networks by ElectromagnetismLike Algorithm, Ph. D. thesis, İnönü University, Malatya, Turkey, 2015

[9] Efrat, A., Har-Peled, S., Mitchell, J.S.B., Approximation algorithms for two optimal location problems in sensor networks, Proceedings, $3^{\text {rd }}$ International Conference on Broadband Communications, Networks and Systems, Boston, Massachusetts, USA, 2005, pp. 767-776

[10] Dhillon, S.S., Chakrabarty, K., Sensor Placement for Effective Coverage and Surveillance in Distributed Sensor Networks, Proceedings, IEEE Wireless Communications and Networking Conference, New Orleans, LA, USA, 2003, pp. 1609-1614 
[11] Toumpis, S., Gupta, G.A., Optimal Placement of Nodes in Large Sensor Networks under a General Physical Layer Model, Proceedings, $2^{\text {nd }}$ IEEE Conference on Sensor and Ad Hoc Communications and Networks, Santa Clara, CA, USA, 2005, pp. 275-283

[12] Biagioni, E.S., Sasaki, G., Wireless Sensor Placement for Reliable and Efficient Data Collection, Proceedings, $36^{\text {th }}$ Annual Hawaii International Conference on System Sciences, Big Island, Hawaii, USA, 2003, Vol. 5, doi:10.1109/HICSS.2003.1174290

[13] Akkaya, K., Younis, M., COLA: A Coverage and Latency aware Actor Placement for Wireless Sensor and Actor Networks, Proceedings, IEEE Vehicular Technology Conference, Montreal, Canada, 2006, pp. 1-5.

[14] Zou, Y., Chakrabarty, K., Sensor deployment and target localization based on virtual forces, Proceedings, $22^{\text {nd }}$ Annual Joint Conference of the IEEE Computer and Communications Societies, San Francisco, CA, USA, 2003, pp. 1293-1303

[15] Li, S., Xu, C., Pan, W., Pan, Y., Sensor deployment optimization for detecting maneuvering targets, Proceedings, $7^{\text {th }}$ International Conference on Information Fusion, Philadelphia, PA, USA, 2005, pp. 1629-1635

[16] Kukunuru, N., Thella, B.R., Davuluri, R.L., Sensor Deployment Using Particle Swarm Optimization, International Journal of Engineering Science and Technology, 2 (2010), 10, pp. 5395-5401.

[17] Wang, X., Wang, S., Ma, J.J., An Improved Co-evolutionary Particle Swarm Optimization for Wireless Sensor Networks with Dynamic Deployment, Sensors, 7 (2007), 3, pp. 354-370.

[18] Ozturk, C., Karaboga, D., Gorkemli, B., Probabilistic Dynamic Deployment of Wireless Sensor Networks by Artificial Bee Colony Algorithm, Sensors, 11 (2011), 6, pp. 6056-6065.

[19] Özdağ, R., Karc1, A., Sensor Node Deployment Based on Electromagnetism-Like Algorithm in Mobile Wireless Sensor Networks, International Journal of Distributed Sensor Networks, 11 (2015), 2, 15 pages, doi.org/10.1155/2015/507967

[20] Özdağ, R., Karc1, A., Probabilistic Dynamic Distribution of Wireless Sensor Networks with Improved Distribution Method based on Electromagnetism-Like Algorithm, Measurement, 79 (2016), pp. 66-76, doi.org/10.1016/j.measurement.2015.09.056

[21] Özdağ, R., A New Meta-heuristic Approach with Dynamic Node Deployment for Area Coverage in Wireless Sensor Networks, Proceedings, $4^{\text {th }}$ International Symposium On Innovative Technologies in Engineering and Science, Alanya, Antalya, Turkey, 2016, pp. 1513-1522

[22] Mirjalili,S., Lewis, A., The Whale Optimization Algorithm, Advances in Engineering Software, 95 (2016), pp. 51-67, doi.org/10.1016/j.advengsoft.2016.01.008

[23] Canayaz, M., Karci, A., Cricket behaviour-based evolutionary computation technique in solving engineering optimization problems, Applied Intelligence, 44 (2016), 2, pp. 362-376, doi.org/10.1007/s 1048

[24] Tanyıldızı, E., Cigal, T., Whale Optimization Algorithms With Chaotic Mapping, Science and Eng. J of Firat Univ., 29 (2017), 1, pp. 309-319. 